\title{
These pretzels are going to make me thirsty tomorrow: Differential development of hot and cool episodic foresight in early childhood?
}

\author{
Caitlin E. V. Mahy ${ }^{1,2 *}$, Julia Grass ${ }^{3}$, Sarah Wagner ${ }^{3}$ and \\ Matthias Kliegel ${ }^{2}$ \\ 'Department of Psychology, University of Oregon, Eugene, Oregon, USA \\ ${ }^{2}$ Department of Psychology, University of Geneva, Switzerland \\ ${ }^{3}$ Department of Psychology, Technische Universität Dresden, Germany
}

\begin{abstract}
The current study examined 3- and 7-year-olds' performance on two types of episodic foresight tasks: A task that required 'cool' reasoning processes about the use of objects in future situations and a task that required 'hot' processes to inhibit a salient current physiological state in order to reason accurately about a future state. Results revealed that 7-year-olds outperformed 3-year-olds on the episodic foresight task that involved cool processes, but did not show age differences in performance on the task that involved hot processes. In fact, both 3- and 7-year-olds performed equally poorly on the task that required predicting a future physiological state that was in conflict with their current state. Further, performance on the two tasks was unrelated. We discuss the results in terms of differing developmental trajectories for episodic foresight tasks that differentially rely on hot and cool processes and the universal difficulties humans have with predicting later outcomes that conflict with current motivational states.
\end{abstract}

In order for individuals to make successful decisions about the future, such as how they will spend their paycheck, they must master the ability to project themselves into the future to understand how they might feel in a given situation. This ability known as episodic foresight is a relatively new field of study in developmental psychology that has recently seen an increase in research interest (Atance, 2008; Atance \& O'Neill, 2001, 2005; Hudson, Mayhew, \& Prabhakar, 2011; Suddendorf, 2010; Suddendorf, Nielsen, \& von Gehlen, 2011; see the 2011 special issue on the development of episodic foresight in Cognitive Development). Episodic foresight is concerned with understanding how we project ourselves into the future to pre-experience an event. This is a critical ability for children to develop as it has implications for decision-making, accuracy in predicting their future thoughts and feelings, as well as anticipating the consequences of their behaviour. For example, a child who fails to anticipate the future may eat too many slices of pizza at a birthday party leaving no room for a delicious slice of cake. The ability to successfully project oneself into the future has implications for a number of cognitive abilities such as planning, prospective memory, and self-control (see Atance \& Jackson, 2009).

\footnotetext{
*Correspondence should be addressed to Caitlin E. V. Mahy, Department of Psychology, University of Oregon, I 7 I 5 Franklin Blvd, Eugene, OR 97403, USA (email: cmahy@uoregon.edu).
} 


\section{The development of episodic foresight in early childhood}

Children as young as 4 years old can successfully answer questions about their future needs (e.g., Atance \& Meltzoff, 2005; Hayne, Gross, McNamee, Fitzgibbon, \& Tustin, 2011; McCormack \& Hanley, 2011; Suddendorf et al., 2011) although this ability continues to develop into the middle childhood years (see Hudson \& Mayhew, 2011; Lagattuta \& Sayfan, 2011). For example, Suddendorf et al. (2011) showed that 4 -year-olds more often made the appropriate choice to secure a solution to a future problem compared to 3-year-olds. Similarly, Russell, Alexis, and Clayton (2010) showed that 3-year-olds had difficulty with questions about what items would be needed in future, whereas 5-year-olds generally performed well. By the age of five, children tend to be fairly proficient in reasoning about future situations across a wide variety of tasks (e.g., Atance \& Meltzoff, 2005; Hudson et al., 2011; McCormack \& Hanley, 2011).

One dimension that episodic foresight tasks differ upon is the inclusion of a current motivational state. The majority of episodic foresight tasks, whether verbal, choice, or location tasks (for a review, see Hudson et al., 2011), require children to reason about the future in the absence of any current manipulation of affective or motivational states (although delay of gratification and other savings tasks do have a motivational future component but lack a strong current affective or motivational internal state that conflicts directly with the future state; Atance \& Jackson, 2009; Metcalf \& Atance, 2011). One task developed by Atance and Meltzoff (2006), however, required children to reason about a future physiological state (i.e., hunger or thirst) while they were currently experiencing a physiological state (i.e., thirst) that conflicted with their usual future preference. During this task, even 5-year-olds had difficulty anticipating their future preference for pretzels in the face of the conflicting current state of thirst. The motivational aspect of a task may be an important contributor to the development of children's episodic foresight, yet it has not been carefully studied.

\section{A hot/cool framework}

A hot/cool framework has been applied to other areas of Cognitive Development, such as executive function, delay of gratification, and relational processing (e.g., Bunch \& Andrews, 2012; Carlson, Davis, \& Leach, 2005; Hongwanishkul, Happaney, Lee, \& Zelazo, 2005; Metcalfe \& Mischel, 1999; Zelazo \& Müller, 2002; Zelazo, Qu, \& Müller, 2005). This framework describes tasks that rely more on 'cool' cognitive processes and those that rely more on 'hot' affective or motivational processes. Whether a task engages relatively cool or hot processes is determined by the nature of the task including its stimuli, feedback, as well as rewards. It is important to note that whether a task relies more on hot or cool processes is dimensional rather than absolute; even tasks that involve relatively hot processes (e.g., delay of gratification) also involve the operation of cool processes (e.g., working memory and inhibition).

Further supporting the distinction between executive tasks that rely more on hot or cool processes is that the hot and cool aspects of tasks differentially rely on particular neural regions. Cool aspects of tasks tend to recruit dorsal lateral prefrontal cortex (DL-PFC), whereas hot aspects tend to recruit ventromedial prefrontal cortex (VM-PFC) and orbitofrontal cortex (Bunch \& Andrews, 2012; Metcalfe \& Mischel, 1999; Zelazo \& Müller, 2002). We propose that a hot/cool framework may be useful to apply to episodic foresight tasks and may yield novel predictions for its development over childhood. 
The development trajectories of hot and cool processes remain unclear, as research has revealed heterogeneous findings. On the one hand, a recent study showed that performance on executive tasks relying on cool processes develops rapidly in early childhood, whereas executive tasks relying on hot processes seem to be more difficult and show an extended developmental trajectory into later childhood (e.g., Prencipe et al., 2011). In much younger children, Prencipe and Zelazo (2005) showed that 3-year-olds, although capable of making adaptive decisions for others, have difficulty overcoming interference from their own desires for immediate gratification. Taken together, these studies suggest that young children still struggle with tasks with an affective or motivational component, whereas they demonstrate better performance on cognitive tasks lacking hot aspects.

On the other hand, several researchers have suggested that young children may rely more on the early developing systems that support the operation of hot processes compared to those requiring cool processes with a shift to engaging cool processes that support performance on more cognitive tasks over development (e.g., Garon, Longard, Bryson, \& Moore, 2012; Metcalfe \& Mischel, 1999). Similarly, Hongwanishkul et al. (2005) showed that executive function tasks involving decision-making in an affective context (children's gambling task and delay of gratification) tended to develop earlier than executive tasks indexing visual working memory and cognitive flexibility lacking an affective context (self-ordered pointing task and dimensional change card sort) in preschool-aged children. In addition, 3- and 4-year-olds' flexible rule use has been shown to benefit from the use of emotional compared to non-emotional stimuli (e.g., Qu \& Zelazo, 2007), suggesting that younger children's task performance may be more influenced by the use of affective material. Moreover, Bunch and Andrews (2012) found that 4-year-olds performed better on tasks relying on hot processes (decision-making, flexible learning, and social understanding) than on tasks relying on cool processes (relational understanding, conceptual understanding, and cognitive flexibility), but that by 6-years of age, this pattern was reversed. Neural evidence shows that VM-PFC (associated with hot executive processes) develops earlier than DL-PFC (associated with cool executive processes; e.g., Gogtay et al., 2004; Orzhekhovskaya, 1981). Taken together, it is unclear what the exact developmental trajectories of hot and cool processes are, as it seems like performance on tasks relying on hot processes may develop earlier than performance on task relying on cool processes (Hongwanishkul et al., 2005; Metcalfe \& Mischel, 1999), yet hot processes may then mature more slowly (Prencipe et al., 2011). In sum, the current literature provides mixed evidence on the relative trajectories of hot and cool executive processes. Finally, the question of whether the hot and cool processes show similar developmental patterns in other areas of cognition remains open.

The current study will attempt to apply a hot/cool framework to the area of episodic foresight and will examine whether there are different trajectories episodic foresight tasks that rely more on hot or cool processes (as has been found in the executive function literature). For the purposes of the current study, hot processing in episodic foresight tasks involve self-projection into future scenarios that have an affective component (e.g., episodically projecting oneself into a situation that is emotional or projecting while a current motivational state conflicts with accurate future thinking), whereas cool processes in episodic foresight tasks involve self-projection that relies on cognitive reasoning and may rely more on semantic scripts (e.g., task that involve episodic projection of the self into the future in neutral situations that lack an emotional or motivational component). 
Many episodic foresight tasks present somewhat familiar scenarios to children and require them to reason about future situations without any need to consider current motivation states (e.g., Atance \& Jackson, 2009; Atance \& Meltzoff, 2005). One example of such a task is the Picture-Book task developed by Atance and Meltzoff (2005) that requires children to imagine going to a particular location the next day (e.g., mountains) and asks them to choose which item they should bring and to explain why they should bring that item. The correct item is one that functionally satisfies a future physiological state (e.g., bringing lunch to the mountains to satisfy the state of hunger). The assumption is that in order to chose the correct answer, lunch, children must imagine being in the mountains in future and recognize that they might be hungry. Therefore, children are asked to reason about a future physiological state in the absence of a current motivational state that might conflict with it. In this way, such tasks may be considered as relying on cool future thinking processes, as they do not rely on affectively salient information. Further, there is no current state (such as hunger or thirst) that must be disengaged from in order to make an appropriate future judgement.

Children show improvement on this particular episodic foresight (Picture-Book) task during the preschool years (Atance \& Meltzoff, 2005; Atance \& O'Neill, 2001; Mahy \& Moses, 2013) potentially due to increases in script-based knowledge gained from experience with similar events as well as advances in episodic projection perhaps gained from practice in anticipating and imagining the future. Although children have a rich repertoire of script-based knowledge from a young age (Hudson, Shapiro, \& Sosa, 1995), 3 -year-olds tend to struggle with tasks such as the Picture-Book task because they rely too heavily upon associative links rather than causal relations when making their item choice. This error leads children to choose the item that is semantically related to the situation instead of the item that will satisfy the future physiological state likely to arise in that situation (Atance \& Meltzoff, 2005). By the time children reach 4 or 5 years old, however, sophisticated planning and anticipatory behaviours seem to be in place (Atance \& O'Neill, 2001). Thus, the preschool years are a period of rapid development in tasks that rely on cool processes involved in episodic foresight with the end result of a seemingly solid understanding of the future by around 5 years old, accomplished by the use of script-based knowledge and episodic future projection (e.g., Atance \& Meltzoff, 2005).

In contrast, episodic foresight tasks that require children to make judgements about their own future states via projection of the self into the future in the face of affectively salient information rely on hot processes. The standard delay of gratification paradigm is an example of such a task. Children must forgo immediately gratification to benefit their future selves. Such tasks pit current and future desires against each other; however, a more powerful way to engage hot processes may be to directly manipulate the current state in order to make decisions about the future especially difficult. A particularly effective way to ensure that children are engaging in self-projection relying on hot processing is to use tasks in which children's current motivational state or desire conflicts with a future state. These tasks by definition require children to project themselves into the future in an episodic manner, as children will perform poorly if they rely on their current state to make future judgements. Atance and Meltzoff (2006) developed an episodic foresight task that accomplished just that. The so-called Pretzel task pits children's general preferences (for pretzels over water) against their current preference after consuming many salty pretzels (water over pretzels). In this task, children were first asked whether they would prefer pretzels or water with the majority of children reporting a preference for pretzels. Then, children were offered an 
opportunity to eat as many pretzels as they wished. After children had consumed several pretzels, children were asked the key episodic foresight question of what would they prefer tomorrow: Water or pretzels? One would expect children to respond pretzels given that children initially preferred pretzels to water. However, if children were unable to disengage from their current state of thirst (a salient current motivational state) to project themselves into the future, they would likely answer that they would prefer water tomorrow. The results of the study showed that 3- to 5-year-olds mostly replied that they would prefer water rather than pretzels the next day, indicating that they failed to appropriately project themselves into the future. This task requires episodic projection to escape the physiological-based desires of the current self, so it is a task that relies on hot processes in that it requires dealing with conflicting motivational states of the current and future self. Other research corroborates that 3-year-olds are impeded by situations that require imagining a future state that is in direct conflict with a current state (Thompson, Barresi, \& Moore, 1997). Interestingly, this so-called 'empathy gap' between the current and future self may be a universal problem in human cognition that affects both children's and adults' episodic foresight (see Gilbert, Gill, \& Wilson, 2002; Loewenstein \& Schkade, 1999; Wilson \& Gilbert, 2003).

Interestingly, performance on the Pretzel task (an episodic foresight task relying on hot processes) did not reveal age differences between 3- and 5-year-old children (Atance \& Meltzoff, 2006), in contrast to most episodic foresight tasks that rely on cool processes, that show developmental improvements during this period (e.g., Atance \& Meltzoff, 2005; Russell et al., 2010; Suddendorf et al., 2011). Given research showing that even adults may struggle with considering a future state in the face of salient current information (e.g., Wilson \& Gilbert, 2003), it may be that performance on tasks requiring hot episodic foresight processes develops more slowly and is not fully developed by the end of the preschool period, in contrast to performance on episodic foresight tasks requiring cool processing.

The body of research on episodic foresight development has had a heavy focus on the preschool years (e.g., Atance \& Jackson, 2009; Busby \& Suddendorf, 2005; Metcalf \& Atance, 2011; Suddendorf \& Busby, 2005) with much less work examining how it develops in early and middle childhood (although see Hudson \& Mayhew, 2011; Lagattuta \& Sayfan, 2011). Without examining children over 5 years old, our knowledge about the developmental trajectory of episodic foresight tasks that rely differentially on hot or cool processes is limited. To the best of our knowledge, no study has attempted to contrast the development of tasks that rely on hot and cool episodic foresight processes in childhood.

The current study will be the first to examine the development of performance on episodic foresight tasks that rely differentially on hot and cool processes in 3- and 7-year-old children. Importantly, this age range bridges the preschool and school period extending past studies to yield a more comprehensive developmental picture of episodic foresight ability across early childhood. We expect that a representative episodic foresight task that relies on cool processing will show rapid improvement in this period, consistent with the past literature (see Hudson et al., 2011 for a review). Given the prediction that overcoming a current salient state to reason about the future is a universal problem, as well as the previous finding of no developmental improvements between 3-and 5-year-olds on the Pretzel task (Atance \& Meltzoff, 2006), we predict that performance on a representative episodic foresight task that relies on hot processes may show less age-related improvement in early childhood. 


\section{Method}

\section{Participants}

Participants were eighteen 3-year-olds (nine girls; $M=3.32, S D=0.44$ ) and eighteen 7 -year-olds (nine girls; $M=7.43, S D=0.47$ ). All children had comparable mental abilities, assessed with the subtest 'information' of the German version of the WPPSI-III (Ricken, Fritz, Schuck, \& PreuB, 2007) for 3-year-olds and the same subtest of the German version of the WISC-III (Tewes, Rossmann, \& Schallenberger, 1999) for 7-year-olds. Children of both age groups had scores in the average to superior range (3-year-olds: $M=12.4, S D=2.5$; 7-year-olds: $M=10.4, S D=2.5$ ). Participants were recruited from the Psychology Department's participant database and flyer advertisements on campus.

\section{Measures}

Future understanding check

Children's conception of the term 'tomorrow' was assessed using a stuffed animal because both episodic foresight tasks relied on the concept and past work indicated that 3 -year-olds have some difficulty with the concept in particular (see Atance \& Meltzoff, 2006). Children were told about a stuffed animal that wanted to 'eat noodles tomorrow' and wanted to 'go to the zoo tomorrow'. The child had to answer whether the stuffed animal wanted to eat noodles/go to the zoo: on the same day, the day before, or the following day. All children answered correctly 'the following day', indicating that they at least had a basic understanding of the meaning 'tomorrow'.

\section{Picture-Book task}

This episodic foresight task was adapted from Atance and Meltzoff (2005). The order of stimulus presentation was switched so that children were presented with one item first and then had to choose one of three photographs of different situations where they could imagine using the given item the next day. This change in stimulus presentation order was made to make the task more concrete for young children so they could rely on the object's potential use to satisfy a future need. In this task, children had to select the appropriate location where they might use the item in future requiring some future projection. The items presented to children were a Band-Aid ${ }^{\mathrm{TM}}$, sunglasses, and a present. For each of these items, the same three photographs were used (rocky stream, beach, and birthday party). After children had selected the scenario in which to use the item, they were asked to explain why they would use the item in that situation. These verbal explanations were scored from 0 to 2 based on how future-oriented their answers were with a 0 for no answer or an irrelevant answer making no reference to the future, 1 for an answer based on a simple association without explicit reference to the future (e.g., 'there is a sun'), and 2 for an answer that made reference to the future by the use of a self-referent (e.g., 'I') or a corresponding state term (e.g., 'hurt'). Explanations were coded independently by two individuals (Cohen's $\kappa=0.86$ ), and any discrepancies were resolved through discussion. The final score for the Picture-Book task combined the number of correct scenarios chosen (out of 3) and children's explanation for each scenario chosen (out of 6) resulting in a score ranging from 0 to 9. 
Pretzel task

This task was adapted from Atance and Meltzoff (2006). Initially, children were asked whether they liked pretzels and all children replied 'yes'. Children were told that they could eat as many pretzels as they liked while the experimenter read a story for about 5 min. A bowl of 30 pretzels was placed next to children, and it was emphasized that eating pretzels was only allowed until the end of the story. After the story was finished, children were asked what they would prefer to eat the next day (the key episodic foresight question): Some water or some pretzels. These options were counterbalanced in order to control for any response bias. The correct answer was considered to be pretzels as they are generally more attractive to children (based on the results of Atance \& Meltzoff, 2006) than water. The incorrect answer was water, as the children were currently thirsty (after eating many salty pretzels), but would likely not be thirsty the next day and would once again prefer pretzels to water. In order to check for level of thirst, the number of pretzels that children ate was counted. Children were given a score of 1 if they answered pretzels and 0 if they answered water.

\section{Procedure}

Children received the tasks in the following fixed order, as is convention in individual difference research: the future understanding check, the 'information' subscale of the German version of the WPPSI-III or of the WISC-III, the Picture-Book task, and the Pretzel task. The university's ethics committee approved all procedures.

\section{Results}

\section{Picture-Book task}

Seven-year-olds $(M=7.61, S D=1.33)$ performed better than 3 -year-olds $(M=1.50$, $S D=1.29)$ on the Picture-Book task, $t(34)=13.94, p<.001$, Cohen's $d=4.66$. When performance for scenario choices was compared to explanations, it was revealed that 3-year-olds performed worse than 7-year-olds both in choosing the correct scenario in which to use the given item the next day and in providing a future-related explanation for the item's use in a particular location, $t \mathrm{~s}(34)>9.71$, $p \mathrm{~s}<.001$, Cohen's $d \mathrm{~s}>3.24$.

\section{Pretzel task}

Overall, fewer children made the correct response of 'pretzels' (12/36) than would be expected by chance, $\chi^{2}(1, N=36)=4.0, p=.05$. There was no difference in 3 - and 7 -year-olds' performance on the Pretzel task $(p=1.0)$. In fact, exactly a third $(6 / 18)$ of both 3- and 7-year-olds provided the correct response of 'pretzels', whereas two-thirds of children answered that they would prefer water the next day. This cannot be explained by a dislike of pretzels children ate a fair number $(M=10.44, S D=5.95)$ that was significantly greater than zero, $t(35)=10.53, p<.001$, and all children indicated that they liked pretzels at the beginning of the study. The number of pretzels eaten was unrelated to whether children chose water or pretzels for the next day. 


\section{Comparison between the two episodic foresight tasks}

In order to compare performance between the two future thinking tasks, we calculated the percentage scores on the Picture-Book and Pretzel tasks (Figure 1). A 2 (age: 3- vs. 7 -year-olds) $\times 2$ (task type: Picture-Book vs. Pretzel task) repeated-measures ANOVA revealed a significant effect of age overall with 7-year-olds outperforming 3-year-olds, $F$ $(1,34)=15.26, p<.001, \eta_{\mathrm{p}}^{2}=.31$. There was also a significant effect of task type such that children performed better on the Picture-Book task compared to the Pretzel task, $F$ $(1,34)=4.45, p=.04, \eta_{\mathrm{p}}^{2}=.12$. These main effects were qualified by a significant interaction between age and task type, $F(1,34)=17.19, p<.001, \eta_{\mathrm{p}}^{2}=.34$. Follow-up comparisons revealed that 7-year-olds outperformed 3-year-olds on the Picture-Book task, $t(34)=13.94, p<.001$, Cohen's $d=4.66$, but there was absolutely no age difference in performance on the Pretzel task, $t(34)=.00, p=1.0$, Cohen's $d=0.00$.

In order to control for the possibility that differences in children's performance on these two episodic foresight tasks were due to the Picture-Book task's multiple trials or high verbal demand (verbal explanations of their choice) compared to the Pretzel task's single trial and minimal verbal demands, we examined age differences in performance on the single trial item choices in the Picture-Book task. These single trials in the Picture-Book task where children had to select the location in which to use a given object (Band-Aid, sunglasses, present) had minimal verbal demand and were therefore very similar to the single trial of the Pretzel task. Comparison of 3-and 7-year-olds' performance in the single trials of the Picture-Book task revealed a significant effect of age with 7-year-olds outperforming 3-year-olds, $t \mathrm{~s}(34)>4.61, p \mathrm{~s}<.001$, Cohen's $d \mathrm{~s}>1.55$. Therefore, the age effect in the Picture-Book task was not simply due to the verbal demand of the task nor to its multiple trial structure, as these age effects were apparent even in a single trial that had minimal verbal demand.

\section{Relationship between the two episodic foresight tasks}

Although age differences were apparent in the Picture-Book task, but not in the Pretzel task, we sought to examine whether individual differences in performance on the two tasks would exist. One might expect that performance may be positively correlated if both rely on similar episodic foresight processes. Interestingly, results revealed that there was

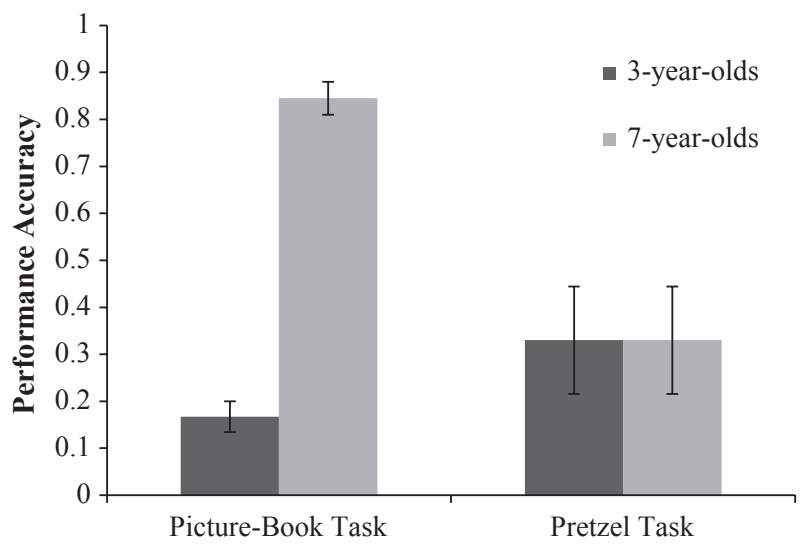

Figure I. Three- and 7-year-olds' accuracy on the Picture-Book and Pretzel tasks. 
no relationship between children's performance on the Picture-Book and Pretzel tasks, $r$ (36) $=.04, p=.81$.

\section{Discussion}

The primary aim of this study was to examine developmental differences between 3-and 7-year-olds' performance on episodic foresight tasks that differentially relied on hot and cool processes. Results revealed a substantial age effect in performance on the Picture-Book task that relied on cool processes, but no age differences whatsoever in performance on the Pretzel task that relied on hot processes. Importantly, these differences were not likely due to superficial differences in the number of trials or verbal demands between the two tasks as age differences remained in the single trials of the Picture-Book task that had extremely low verbal requirements. These results suggest that children's performance on episodic foresight tasks that rely on reasoning in the absence of motivational content develops earlier than tasks that involve salient motivational information that must be overcome in order to project one's future states. These findings are similar to Prencipe et al.'s (2011) with 8- to 15-year-old children that suggested that performance on executive tasks relying on hot processes were later and slower to develop compared to performance on executive tasks relying on cool processes. However, research with preschool-aged children generally shows that performance on executive control tasks requiring hot processes develops earlier than performance on executive control tasks requiring cool processes (Hongwanishkul et al., 2005; Metcalfe \& Mischel, 1999) and that motivational information facilitates performance in this age range (Qu \& Zelazo, 2007). Thus, the current results may be specific to the episodic foresight tasks used in the study. Additionally, episodic foresight may represent a different set of cognitive processes than those used for executive function so therefore may not show the same relative trajectories of performance on tasks that rely on hot and cool processing during the preschool years.

The developmental trajectory of performance on the Picture-Book task in the current study is consistent with much of the literature suggesting that by the time children reach 4 or 5 years old, their episodic foresight (at least in tasks lacking a motivational or affective component) is fairly accurate compared to 3-year-olds (e.g., Atance \& Meltzoff, 2005; Hayne et al., 2011; McCormack \& Hanley, 2011; Suddendorf, 2010). Further, the current results extend past research to show that the Picture-Book task is age appropriate for 7 -year-olds, as they did not perform at ceiling levels, although their performance was high (>80\%).

Remarkably, there was absolutely no difference in 3- and 7-year-olds' performance on the Pretzel task (that relied on hot processes), indicating that even by 7 years of age when episodic foresight is fairly sophisticated when it relies on cool processes (as indexed by performance on the Picture-Book task), children still struggle with accurately representing a future desire when faced with salient physiological state. The lack of age effect on performance of the Pretzel task is consistent with Atance and Meltzoff's (2006) identical finding with 3-and 5-year-olds. Other episodic foresight tasks could be modified to include a current state that conflicts with future desires, such as having children perform a delay of gratification task with food rewards when they are very hungry versus when they are satiated. Currently, the Pretzel task is rather unique from other episodic foresight tasks that rely on hot processing (such as delay of gratification-type tasks), as it is the only task that has been used that directly pits a current state against a future state.

Overcoming an emotionally or physiologically salient situation in order to simulate the future has been suggested to be difficult even for adults as evident in adult's errors in 
affective forecasting (Wilson \& Gilbert, 2003). This study supports the idea that ignoring a current physiological state to make an accurate prediction about a future state may develop much later in childhood or may be indicative of a more general human cognitive error that even adults would struggle with (Atance \& Meltzoff, 2006).

Although it is tempting to conclude that 7-year-old children are unable to project themselves into the future in the face of a salient current motivational state, it is possible that they do project themselves successfully into the future but simply have poor accuracy in their predictions. The distinction between an inability to engage in episodic foresight and inaccuracy during episodic future projection is important, as it is easy to assume that low performance represents a real failure of episodic foresight. Rather than deficits in episodic foresight, it is possible that the inhibitory demand of the episodic foresight task relying on hot processes compared to the task relying on cool processes led to poor performance. Past studies, however, have documented substantial increases in inhibitory control between 3- and 7-year-olds (e.g., Carlson, 2005) so it seems unlikely that poor inhibition in 7-year-olds was responsible for this effect, although it may have played a role in the performance of 3-year-olds. Nonetheless, future work should examine the role of inhibition in various types of episodic foresight tasks that vary in the extent to which current motivational states that must be inhibited in order to perform well on the task.

Performance on the two episodic foresight tasks in the current study did not correlate with each other, suggesting that individual differences in episodic foresight may not be stable across all types of future thinking tasks. This lack of relationship supports the distinction between tasks that differentially rely on hot or cool processing. We suggest that lack of relationship in performance was due to the two tasks tapping different aspects of episodic foresight: One relying on episodic foresight requiring reasoning and perhaps the use of semantic scripts and the other relying on episodic foresight in the face of a highly motivating and salient state. It is possible, however, that other differences between the two tasks such as the amount of inhibition necessary to make a correct response contributed to the interaction between age and task type.

This study presents preliminary evidence of dissociated developmental trajectories of episodic foresight tasks that rely on hot and cool processes, but future studies need to replicate this finding with a greater number of tasks and with a larger sample size. It is important to note, however, that the current data did not suffer from a lack of power as there was absolutely no difference in performance on the Pretzel task, nor did task performance show ceiling or floor levels, indicating that our effects were not due to psychometric problems with the specific tasks employed.

We recognize that the current study was an initial attempt to examine developmental differences between two types of episodic foresight tasks that had some limitations. One limitation was the use of only two episodic foresight tasks. There is a possibility that the two tasks used in the current study could have differed on dimensions other than their affective salience that could have contributed to their differing developmental trajectories. We argue that the affective aspect of the current physiological state in the Pretzel task led to lower levels of performance rather than superficial differences such as the number of trials or verbal demands.

In conclusion, the present study is first to apply the hot/cool framework to the area of children's episodic foresight and to examine the developmental trajectory of episodic foresight tasks that rely on hot and cool processes in children bridging the preschool and early school years. Results revealed a clear developmental dissociation with age-related improvements in an episodic foresight task that relied on cool reasoning processes, but no 
age differences in performance in an episodic foresight task that relied on hot affective processes. Our finding suggests that episodic foresight tasks that involve conflicting current and future states may still pose a challenge for older children and perhaps adults, in contrast to episodic foresight tasks with no affective component, which may be fairly well developed by 5-year-olds. This study is an initial indication of differing developmental trajectories within episodic foresight tasks and represents a promising area for future inquiry.

\section{Acknowledgements}

Preparation of this manuscript was supported by a Swiss Government Scholarship to CEVM.

\section{References}

Atance, C. M. (2008). Future thinking in young children. Current Directions in Psychological Science, 17, 295-298. doi:10.1111/j.1467-8721.2008.00593.x

Atance, C. M., \& Jackson, L. K. (2009). The development and coherence of future-oriented behaviors during the preschool years. Journal of Experimental Child Psychology, 102, 379-391. doi:10. 1016/j.jecp.2009.01.001

Atance, C. M., \& Meltzoff, A. N. (2005). My future self: Young children's ability to anticipate and explain future states. Cognitive Development, 20,341-361. doi:10.1016/j.cogdev.2005.05.001

Atance, C. M., \& Meltzoff, A. N. (2006). Preschoolers' current desires warp their choices for the future. Psychological Science, 17, 583-587. doi:10.1111/j.1467-9280.2006.01748.x

Atance, C. M., \& O'Neill, D. K. (2001). Episodic future thinking. Trends in Cognitive Sciences, 5, 533-539. doi:10.1016/S1364-6613(00)01804-0

Atance, C. M., \& O'Neill, D. K. (2005). The emergence of episodic future thinking in humans. Learning and Motivation, 36, 126-144. doi:10.1016/j.Imot.2005.02.003

Bunch, K. M., \& Andrews, G. (2012). Development of relational processing in hot and cool tasks. Developmental Neuropsychology, 37, 134-152. doi:10.1080/87565641.2011.632457

Busby, J., \& Suddendorf, T. (2005). Recalling yesterday and predicting tomorrow. Cognitive Development, 20, 362-372. doi:10.1016/j.cogdev.2005.05.002

Carlson, S. M. (2005). Developmentally sensitive measures of executive function in preschool children. Developmental Neuropsychology, 28, 595-616. doi:10.1207/s15326942dn2802_3

Carlson, S. M., Davis, A. C., \& Leach, J. G. (2005). Less is more: Executive function and symbolic representation in preschool children. Psychological Science, 16, 609-616. doi:10.1111/j. 1467-9280.2005.01583.x

Garon, N. M., Longard, J., Bryson, S. E., \& Moore, C. (2012). Making decisions about now and later: Development of future-oriented self-control. Cognitive Development, 27, 314-322. doi:10. 1016/j.cogdev.2012.05.003

Gilbert, D. T., Gill, M. J., \& Wilson, T. D. (2002). The future is now: Temporal correction in affective forecasting. Organizational Behavior and Human Decision Processes, 88, 690-700. doi:10. 1006/obhd.2001.2982

Gogtay, N., Giedd, J. N., Lusk, L., Hayashi, K. M., Greenstein, D., Vaituzis, A. C., . . Thompson, P. M. (2004). Dynamic mapping of human cortical development during childhood through early adulthood. Proceedings of the National Academy of Sciences of the United States of America, 101, 8174-8179. doi:10.1073/pnas.0402680101

Hayne, H., Gross, J., McNamee, S., Fitzgibbon, O., \& Tustin, K. (2011). Episodic memory and episodic foresight in 3- and 5-year-old children. Cognitive Development, 26, 343-355. doi:10. 1016/j.cogdev.2011.09.006

Hongwanishkul, D., Happaney, K. R., Lee, W. S., \& Zelazo, P. D. (2005). Assessment of hot and cool executive function in young children: Age-related changes and individual differences. Developmental Neuropsychology, 28, 617-644. doi:10.1207/s15326942dn2802_4 
Hudson, J. A., \& Mayhew, E. M. Y. (2011). Children's temporal judgments for autobiographical past and future events. Cognitive Development, 26, 331-342. doi:10.1016/j.cogdev.2011.09.005

Hudson, J. A., Mayhew, E. M., \& Prabhakar, J. (2011). The development of episodic foresight: Emerging concepts and methods. Advances in Child Development and Behavior, 40, 95-137. doi:10.1016/B978-0-12-386491-8.00003-7

Hudson, J. A., Shapiro, L. R., \& Sosa, B. B. (1995). Planning in the real world: Preschool children's scripts and plans for familiar events. Child Development, 66, 984-998. doi:10.1111/j. 1467-8624.1995.tb00917.x

Lagattuta, K. H., \& Sayfan, L. (2011). Developmental changes in children's understanding of future likelihood and uncertainty. Cognitive Development, 26, 315-330. doi:10.1016/j.cogdev.2011. 09.004

Loewenstein, G., \& Schkade, D. (1999). Wouldn't it be nice? Predicting future feelings. In D. Kahneman, E. Diener \& N. Schwarz (Eds.), Well-being: The Foundations of Hedonic Psychology (pp. 85-105). New York: Russell Sage Foundation.

Mahy, C. E. V., \& Moses, L. J. (2013, April). The role of cognitive monitoring in children's remembering. Symposium session presented at the biennial meeting of the Society for Research in Child Development, Seattle, WA.

McCormack, T., \& Hanley, M. (2011). Children's reasoning about the temporal order of past and future events. Cognitive Development, 26, 299-314.

Metcalf, J. L., \& Atance, C. M. (2011). Do preschoolers save to benefit their future selves? Cognitive Development, 26, 371-382. doi:10.1016/j.cogdev.2011.09.003

Metcalfe, J., \& Mischel, W. (1999). A hot/cool-system analysis of delay of gratification: Dynamics of willpower. Psychological Review, 106, 3-19. doi:10.1037/0033-295X.106.1.3

Orzhekhovskaya, N. S. (1981). Fronto-striatal relationships in primate ontogeny. Neuroscience $\&$ Behavioral Physiology, 11, 379-385. doi:10.1007/BF01184205

Prencipe, A., Kesek, A., Cohen, J., Lamm, C., Lewis, M. D., \& Zelazo, P. D. (2011). Development of hot and cool executive function during the transition to adolescence. Journal of Experimental Child Psychology, 108, 621-637. doi:10.1016/j.jecp.2010.09.008

Prencipe, A., \& Zelazo, P. D. (2005). Development of affective decision making for self and other evidence for the integration of first-and third-person perspectives. Psychological Science, 16, 501-505. doi:10.1111/j.0956-7976.2005.01564.x

Qu, L., \& Zelazo, P. D. (2007). The facilitative effect of positive stimuli on 3-year-olds' flexible rule use. Cognitive Development, 22, 456-473. doi:10.1016/j.cogdev.2007.08.010

Ricken, G., Fritz, A., Schuck, K. D., \& PreuB, U. (2007). HAWIVA-III. Manual zur Testentwicklung und interpretation [Manual for Test Development and Interpretation]. Bern, Switzerland: Hogrefe AG.

Russell, J., Alexis, D., \& Clayton, N. (2010). Episodic future thinking in 3- to 5-year-old children: The ability to think of what will be needed from a different point of view. Cognition, 114, 56-71. doi:10.1016/j.cognition.2009.08.013

Suddendorf, T. (2010). Linking yesterday and tomorrow: Preschoolers' ability to report temporally displaced events. The British Journal of Developmental Psychology, 28, 491-498. doi:10.1348/ 026151009X479169

Suddendorf, T., \& Busby, J. (2005). Making decisions with the future in mind: Developmental and comparative identification of mental time travel. Learning and Motivation, 36, 110-125. doi:10.1016/j.lmot.2005.02.010

Suddendorf, T., Nielsen, M., \& von Gehlen, R. (2011). Children's capacity to remember a novel problem and to secure its future solution. Developmental Science, 14, 26-33. doi:10.1111/j. 1467-7687.2010.00950.x

Tewes, U., Rossmann, P., \& Schallenberger, U. (1999). Hamburg-Wechsler intelligence test for children III (HAWIK-III). Bern, Switzerland: Huber [in German].

Thompson, C., Barresi, J., \& Moore, C. (1997). The development of future-oriented prudence and altruism in preschoolers. Cognitive Development, 12, 199-212. 
Wilson, T. D., \& Gilbert, D. T. (2003). Affective forecasting. Advances in Experimental Social Psychology, 35, 345-411. doi:10.1016/S0065-2601(03)01006-2

Zelazo,P.D.,\&Müller,U.(2002).Executivefunctionintypicalandatypicaldevelopment.InU.Goswami (Ed.), Handbook of childhood cognitive development (pp. 445-469). Oxford, UK: Blackwell.

Zelazo, P. D., Qu, L., \& Müller, U. (2005). Hot and cool aspects of executive function: Relations in early development. In W. Schneider, R. Schumann-Hengsteler \& B. Sodian (Eds.), Young children's cognitive development: Interrelationships among executive functioning, working memory, verbal ability, and theory of mind (pp. 71-93). Mahwah, NJ: Lawrence Erlbaum.

Received 4 June 2013; revised version received 10 September 2013 